\title{
Superconductivity, Superfluidity and Zero-Point Oscillations
}

\author{
Boris V. Vasiliev \\ Independent Researcher, Dubna, Russia \\ Email: bv.vasiliev@yandex.com
}

How to cite this paper: Vasiliev, B.V. (2018) Superconductivity, Superfluidity and Zero-Point Oscillations. Journal of Modern Physics, 9, 315-319.

https://doi.org/10.4236/jmp.2018.93022

Received: September 12, 2017

Accepted: January 29, 2018

Published: February 1, 2018

Copyright $\odot 2018$ by author and Scientific Research Publishing Inc. This work is licensed under the Creative Commons Attribution International License (CC BY 4.0).

http://creativecommons.org/licenses/by/4.0/

\begin{abstract}
It is shown that both super phenomena-superconductivity and superfluidity are based on the same mechanism of streamline of zero-point oscillations. Proof of this is the agreement of obtained theoretical estimations with measured data.
\end{abstract}

\section{Keywords}

Superconductivity, Superfluidity, Zero-Point Oscillations

\section{Introduction}

Superconductivity and superfluidity are similar phenomena. Superconductivity can be regarded as superfluidity of electron gas. This suggests that the nature of these phenomena should be based on the same mechanism.

Despite the fact that superconductivity and superfluidity were discovered many decades ago, the physics of these phenomena remains unclear. For a long time, these phenomena were the most mysterious in condensed matter physics.

An important step towards understanding the physics of superconductivity was the discovery of the electron pairing manifested by the quantization of magnetic flux.

The effect of pairing of electrons was pointed out that superconducting carriers should form the ensemble attracted to each other particles. In this ensemble, particles cannot lose energy scattered by defects in the lattice (phonons) if these losses are less than the energy of their mutual attraction.

At about the same time with the effect of quantization of flux, the isotope effect in superconductors was discovered. It seemed that this effect can be considered as evidence of the dominant role of phonons in the appearance of superconductivity. 
Taking this into consideration, L. Cooper showed that the exchange of phonons may lead to pairing of electrons and J. Bardeen, L. Cooper and J. Shriffer have created the microscopic theory of superconductivity (so called the BCS-theory).

However, the phonon mechanism destroyed the hypothetical link between superconductivity and superfluidity. There are no phonons into liquid helium which is able to combine its atoms in a single ensemble.

A similar situation arose at the description of superfluidity. L. D. Landau in his first works devoted to this problem showed that the phenomenon of superfluidity can be understood if we assume existence of the ensemble of helium atoms being in one quantum state and obeys quantum laws. This allowed him to describe the main features of this phenomenon, the temperature dependence of the density of the superfluid phase, the speed of sound, etc. However, no reply was received to the question what physical mechanism leads to the appearing of superfluid ensemble and why the ratio of the transition temperature to the superfluid state to the boiling point almost exactly equal to $1 / 2$ for helium-4, while for helium-3 it about a thousand times smaller.

Such descriptions of superconductivity and superfluidity create a sense of dissatisfaction due to the fact that is not found the overall mechanism of occurrence of these related phenomena.

As shown by decades of research, the BCS-theory quite successfully describes the general features of the phenomenon of superconductivity, but is not able to describe the main characteristics of the individual superconductors.

As shown by later experiments, the assumption that the phonon mechanism is the only possible mechanism of superconductivity was wrong. It has been proven the anharmonicity of zero-point oscillations of ions in the lattice of metals. As a result of this anharmonicity, isotopic substitution leads to a change in the amplitude of the zero-point oscillations of ions, i.e. interatomic distances in the crystal lattice of metal directly depend on the masses of the isotopes. As a result, isotopic substitution effects directly on the Fermi-energy of metal and phonons do not play any role in it.

\section{Superconductivity and Zero-Point Oscillations [1] [2] [3] [4] [5]}

Superfluidity of helium and superconductivity in metals exist at very low temperatures. At this temperature, all motion of particles freeze, except their zero-point oscillations.

Therefore, at very low temperatures, charged particles can interact with each other through the electromagnetic field of their zero-point oscillations.

The ordering of zero-point oscillations at interacting their electromagnetic fields causes an attraction between particles and lowering of energy of the entire ensemble. Theoretical estimates of critical parameters of this process of streamline are in satisfactory agreement with measurements of properties of supercon- 
ductors.

Calculations in the framework of the model of ordering zero-point oscillations show that the critical temperature of superconductors equal to about 10 to the minus 6th degree from Fermi temperature of superconducting metal, which is consistent with measured data (Figure 1).

The critical magnetic field destroys the coherence of zero-point oscillations of electron pairs. This also agrees well with the measurements (Figure 2).

\section{Superfluidity and Zero-Point Oscillations [1] [2] [6]}

A remarkable result, which is uncharacteristic for condensed matter physics, the application of this theory gives to the description of superfluidity. It turns out that some of basic parameters of superfluid liquid depend on universal constants only and therefore they can be calculated with a very high accuracy. The calculation shows that the temperature of zero-point oscillations ordering, below which helium becomes superfluid state, defined by the equation:

$$
T_{c}=\frac{M_{4} c^{2} \alpha^{6}}{3 k} \approx 2.1778 \mathrm{~K}
$$

where $\alpha$ is fine structure constant, $M_{4}$ is mass of helium atom.

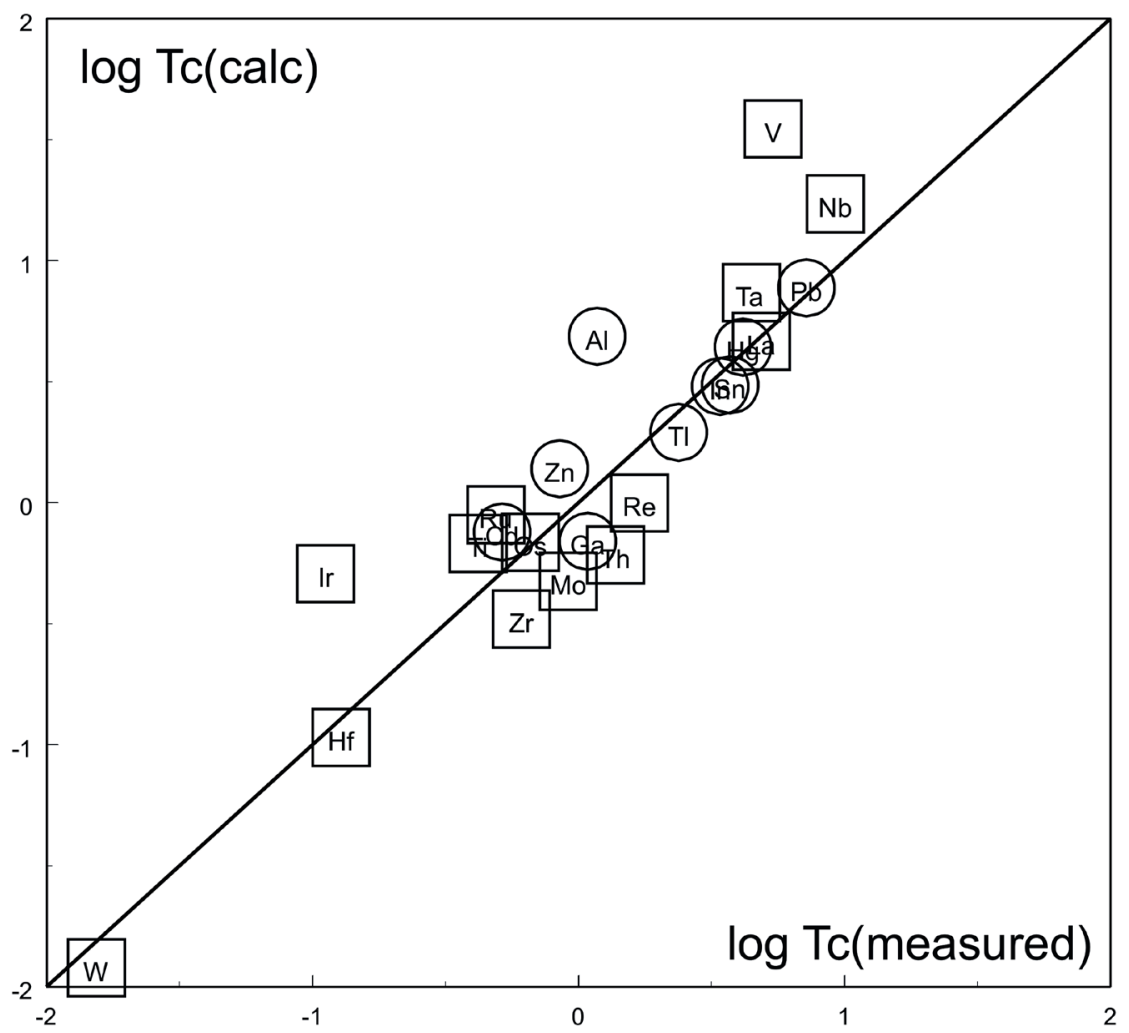

Figure 1. The comparison of the calculated values of critical temperatures of superconductors with measurement data. Circles relate to type-I superconductors, squares show type-II superconductors. On the abscissa, the measured values of critical temperatures are plotted, on ordinate, the calculated estimations are plotted. 


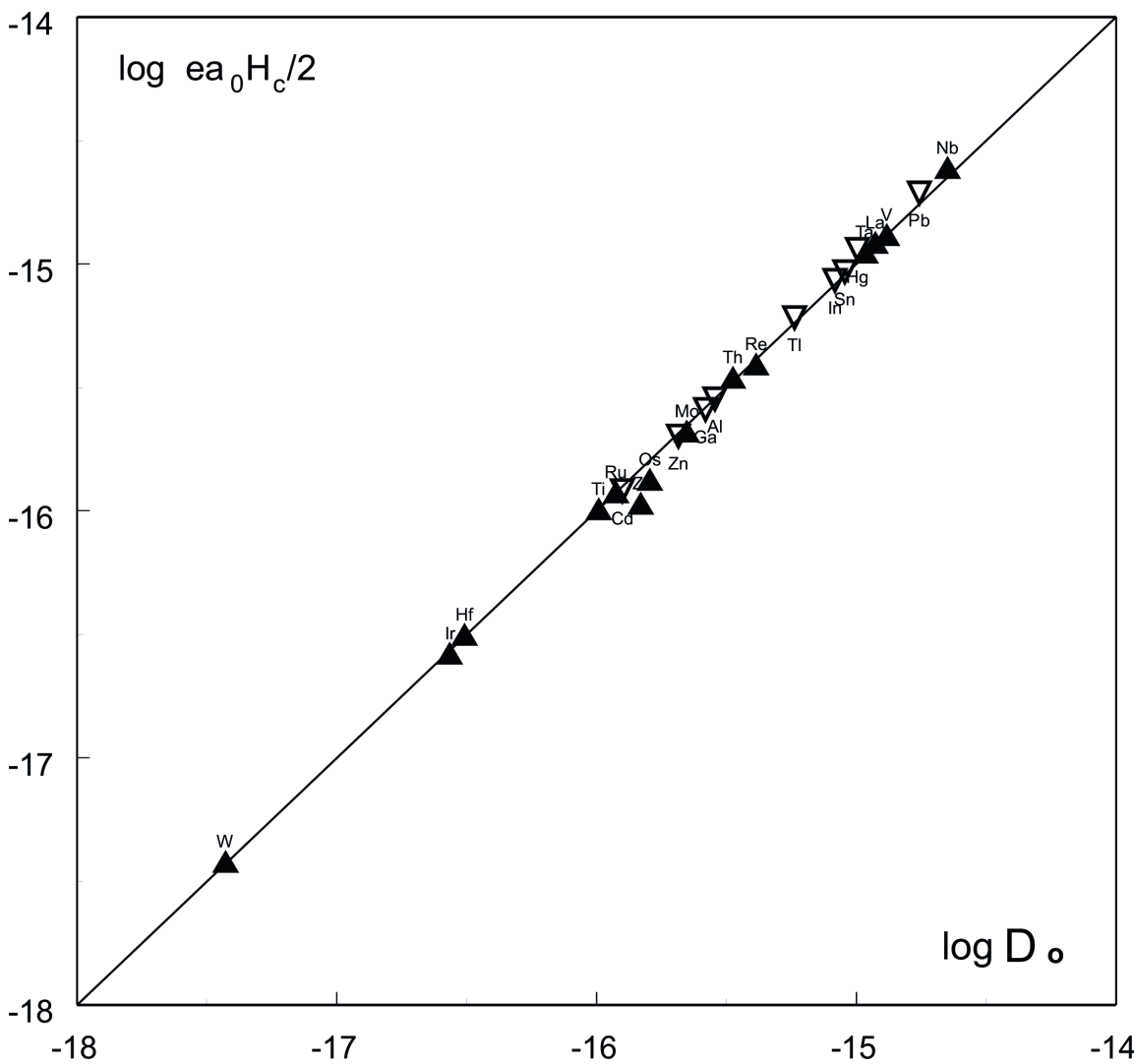

Figure 2. The comparison of the calculated energy of superconducting pairs in the critical magnetic field with the value of the superconducting gap. Here, the following key applies: filled triangles-type-II superconductors, empty triangles-type-I superconductors. On vertical axis-logarithm of the product of the calculated value of the oscillating dipole moment of an electron pair on the critical magnetic field is plotted. On horizontal axisthe value of the gap is shown.

It is in a very good agreement with the measured value of superfluid transition temperature $T_{c}=2.1768 \mathrm{~K}$.

It is also possible to calculate the density of liquid helium in superfluid state. It turns out that the density of particles, also depends on the ratio of fundamental constants only:

$$
\gamma_{4}=\frac{\alpha^{2}}{a_{B}^{3}} \sqrt{\frac{M_{4}^{3}}{2 m_{e}}} \approx 0.1443 \mathrm{~g} / \mathrm{cm}^{3},
$$

where $a_{B}$ is the Bohr radius, $m_{e}$ is electron mass.

This agrees well with measured density of liquid helium equal to $0.145 \mathrm{~g} / \mathrm{cm}^{3}$.

It should be emphasized that the above formulas previously were not known.

The model of superfluidity and superconductivity based on the mechanism of zero-point oscillations ordering are considered in detail in [1].

\section{Conclusion}

Thus the consent of result of calculation in the framework of this model with the measurement data clearly shows that both superconductivity and superfluidity 
arise as follow of the work of the same mechanism, both of these related phenomena occur as a result of ordering zero-point oscillations.

\section{References}

[1] Vasiliev, B.V. (2015) Superconductivity and Superfluidity. Science Publishing Group, New York.

http://www.sciencepublishinggroup.com/book/B-978-1-940366-36-4.aspx

[2] Vasiliev, B.V. (2013) Superconductivity, Superfluidity and Zero-Point Oscillations in "Recent Advances in Superconductivity Research". Nova Publisher, New York, 249-280.

[3] Vasiliev, B.V. (2014) Universal Journal of Physics and Application, 2, 22-35.

[4] Vasiliev, B.V. (2012) Physica C: Superconductivity, 483, 233-246.

https://doi.org/10.1016/j.physc.2012.05.007

[5] Vasiliev, B.V. (2011) Physica C: Superconductivity, 471, 277-284.

https://doi.org/10.1016/j.physc.2010.10.010

[6] Vasiliev, B.V. (2014) Universal Journal of Physics and Application, 2, 165-170. 